\title{
Impact of graphene oxide embedded polyethersulfone membranes for the effective treatment of distillery effluent.
}

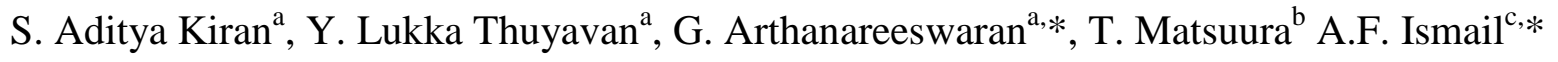

${ }^{a}$ Membrane Research Laboratory, Department of Chemical Engineering, National Institute of Technology, Tiruchirappalli-620015, India

${ }^{\mathrm{b}}$ Department of chemical and Biological Engineering, University of Ottawa, 161 Louis Pasteur St., Ottawa, ON K1N 6N5,Canada.

${ }^{c}$ Advanced Membrane Technology Research Centre (AMTEC), Universiti Teknologi Malaysia, 81310 UTM Skudai, Johor, Malaysia

*Corresponding authors

E-mail address: arthanaree10@yahoo.com (G. Arthanareeswaran), fauzi.ismail@gmail.com (A.F. Ismail). *Corresponding author. Tel: +91431-2503118; Fax: +91431-2500133. 


\section{ABSTRACT}

In the present study, treatment of spent wash from distilleries was studied using graphene oxide (GO) flakes incorporated in polyethersulfone (PES) mixed matrix membranes (MMMs). Two different additives such as poly acrylic acid (PAA) and lithium chloride ( $\mathrm{LiCl}$ ) were also used separately in the casting dope solution of PES/GO. GO TEM analysis revealed that particles are wrinkled sheets-like multilayer structure. Multilayer structures exhibit better intercalate with polymer matrix to produce hybrid nanocomposite membranes. The TGA analysis has shown that GO has good miscibility in PES membrane. Water flux has been improved progressively for the additive combined GO incorporated PES MMMs. Further, contact angle value of the neat PES

membrane showed $70.8^{\circ}$ which was significantly reduced up to $58.4^{\circ}$ for $\mathrm{PES} / \mathrm{GO} / \mathrm{PAA}$ membrane. This ensures that membrane surface hydrophilicity was improved considerably due to the existence of both groups, GO and PAA. Moreover, the membrane performance was evaluated in terms of permeate flux, flux recovery ratio and color removal efficiency for synthetic melanoidin solution as well as distillery spent wash effluent. A maximum of $54 \%$ color removal was observed for distillery spent wash effluent.

Keywords: Graphene oxide, polyethersulfone, melanoidin, membrane characterization and spent wash. 


\section{Introduction}

The rise in demand for distillery products has boosted the economy of Indian market greatly. Distilleries generate $15 \mathrm{~L}$ of high strength wastewater namely dark brown colored molasses spent wash during the production of $1 \mathrm{~L}$ of ethanol. The major chemical constituents of spent wash are polysaccharides, lignin, proteins, melanoidin, fertilizer based inorganic salts and wax [1]. These compounds are major pollutants in the seed germination and eutrophication of water resources respectively [2]. Therefore, it is necessary to treat the distillery spent wash wastewater. The most common method of spent wash treatment are adsorption, coagulation, oxidation, membrane separation and biological mode of anaerobic and aerobic digestion [3]. The prevalent mode of treatment is of biological anaerobic and aerobic digestion which requires time consuming process and need additional treatments [4]. Biopolymer melanoidin is the major pollutant arisen from the distillery effluent and it is hard to decompose by the microorganism $[1,4]$. Among the various technologies, membrane technology has been recommended for the industries to meet the stringent environmental regulations for various wastewater treatment [5]. The membrane filtration has an advantage due to its cost effectiveness, no phase change operation, energy efficient, easy to scale up [5].

Among the membrane material, PES has a desired characteristics of good film property, chemical and mechanical resistant. It also had good miscible properties with hydrophilic additives [6]. However, the intrinsic hydrophobic property leads to major limitation which cause fouling under membrane separation [6]. In order to overcome fouling, researchers have explored mixed matrix membranes (MMMs) in the treatment of various industrial effluents. MMMs are referred to be a heterogeneous composition of both inorganic nanomaterials and base polymer 
[7]. This combination leads in producing prerequisite properties such as enhanced hydrophilicity and better material strength. It ultimately results in the improvement of membrane performance and shelf life of the membrane. The present study focuses on the development of graphene oxide (GO) flakes incorporated in polyethersulfone (PES) mixed matrix membranes for spent wash treatment.

Graphene oxide exhibits various attractive properties such as two dimensional structure, superior electron transport, mechanical stability, hydrophilic nature, promote negative surface charges and better miscibility with polymers [8]. Lee et al. [9] prepared polysulfone/GO membrane for treatment of wastewater in membrane bioreactor. The membrane possesses higher resistance to both antifouling and anti-biofouling properties. Zinadini et al. [10] reported the influence of GO nanoplates on PES/PVP membrane for dye desalination and powder milk filtration. An improvement of membrane flux, and lesser reversible fouling as well as irreversible fouling was observed. Lately, researchers are focusing on the development of novel functionalized GO nanoparticles-polymer nanocomposites for enhancing the biofouling and hydrophilic properties [11]. Therefore, the above studies show that GO has a significant influence on desired membrane properties.

Thus, the present study is attempted for the treatment of distillery effluent using GO incorporated PES membrane. Various researchers also reported an enhancement in the membrane properties using addition of additives like lithium chloride ( $\mathrm{LiCl})$ and polyacrylic acid (PAA) [12]. However, the influence of additives on nanocomposite polymer-GO membrane is also limited. Hence, inorganic and organic additives such as lithium chloride $(\mathrm{LiCl})$ and 
polyacrylic acid (PAA) were also used. Herein the study includes, synthesis of graphene oxide using modified hummers method [13].

- Fabrication of PES/GO MMMs and additives incorporated PES/GO-LiCl, PES/GO-PAA MMMs.

- Prepared membranes were characterized using XRD, SEM, TGA and contact angle analysis.

- Membrane performance was also assessed using both synthetic melanoidin solution and spent wash from a distillery.

\section{Materials and methods}

\subsection{Materials}

Commercial grade PES (Veradale 3000) was procured from Solvay Chemicals (India) Ltd. N, N-dimethyl acetamide (DMAc) and sodium lauryl sulphate (SLS) were purchased from Qualigens Chemicals Ltd., India. D-Glucose, glycine, bovine serum albumin (BSA) and sodium bicarbonate were procured from Merck Chemicals, India Ltd. Lithium chloride ( $\mathrm{LiCl})$ and poly acrylic acid (PAA) were purchased from Merck Chemicals, India Ltd and Sigma-Aldrich Ltd, respectively. Concentrated sulphuric acid, potassium permanganate and hydrochloric acid were bought from Finar Chemicals Ltd., India. All the chemicals are of analytical grade.

\subsection{Preparation and characterization of grapheme oxide nanoparticles}

Graphene oxide nanoparticles were synthesized from graphite powder using modified Hummer's method [13,14]. Initially, $2 \mathrm{~g}$ of SP-1 graphite was grounded with $1 \mathrm{~g}$ of 
sodium chloride to reduce particle size and washed with distilled water and filtered. Thereafter, the filtrate was dissolved in $46 \mathrm{ml}$ of concentrated sulphuric acid for a time period of $8 \mathrm{~h}$. Subsequently, $6 \mathrm{~g}$ of oxidizing agent potassium permanganate was added gradually into the reaction mixture. The temperature was maintained at less than $20^{\circ} \mathrm{C}$ to prevent overheating and explosion. The reaction temperature was ramped up slowly from 35 to $80{ }^{\circ} \mathrm{C}$ for $30 \mathrm{~min}$ and 40 min respectively. The resulting suspension was diluted with $92 \mathrm{ml}$ of water and then heated to $100{ }^{\circ} \mathrm{C}$. Besides, it was diluted with $280 \mathrm{ml}$ of water and treated with $10 \mathrm{ml}$ of $30 \% \mathrm{H}_{2} \mathrm{O}_{2}$ solution. Finally, the mixture was washed through centrifugation and filtration with $5 \%$ of hydrochloric acid and then with water. Then, the synthesized graphene oxide (GO) was dried in vacuum. GO nanoparticles were characterized using transmission electron microscopy (TEM) and XRD. Transmission electron microscopy (TEM) of GO nanoparticles was carried out under $200 \mathrm{kV}$ using JEOL (Model JEM-3010). The crystalline structure of graphene oxide (GO) nanoparticles was studied using X-ray diffractometer (Model D8 advance, Bruker, Germany) using the monochromatic source of $\mathrm{Cu} k \alpha$ radiation. The samples were studied in $2 \theta$ range of $10^{\circ}-80^{\circ}$ under an acceleration voltage of $40 \mathrm{kV}$. As-prepared GO particles was also characterized using Fourier transform infrared spectroscopy (Thermo Scientific Nicolet iS5 FT-IR spectrometer). The spectra were collected in the range of wavelengths from 4000 to $550 \mathrm{~cm}^{-1}$.

\subsection{Preparation of PES/Graphene oxide mixed matrix membranes}

Prior to the preparation of casting dope solution, amorphous PES was dried in a hot air oven at $60{ }^{\circ} \mathrm{C}$ for $8 \mathrm{~h}$. Initially GO oxide was dissolved in DMAc under constant stirring for $2 \mathrm{~h}$ and consequently ultrasonicated for $1 \mathrm{~h}$. Next to that, dried PES polymer was added and allowed to stir for $24 \mathrm{~h}$ at $70{ }^{\circ} \mathrm{C}$. The composition and labeling of neat PES and PES/GO MMMs is given 
in Table 1. Dope solution was again ultrasonicated for $1 \mathrm{~h}$ to attain better dispersion of GO with PES. The obtained homogenous casting solution was allowed to stand for $3 \mathrm{~h}$ to remove air bubbles. After the preparation of dope solution, casting was initiated by pouring the dope solution on the glass plate. Next, the solution was spread over the smooth glass plate with the help of thin film applicator at a thickness of $400 \mu \mathrm{m}$. Delay time of $30 \mathrm{~s}$ was maintained for thin film formation. Subsequently glass plates were immersed in a non-solvent bath containing water at a temperature of $10{ }^{\circ} \mathrm{C}$ for $24 \mathrm{~h}$. It is a rate determining step in membrane formation which depends on the exchange of solvent-non-solvent. The resultant fabricated membranes were washed with double distilled water and stored. The exchange rate can vary corresponding to the additives. In order to study the influence of additives, $\mathrm{LiCl}$ and PAA were used in MMMs preparation and performance.

\subsection{Characterization of PES/GO mixed matrix membranes MMMs}

Contact angle of the prepared membranes was measured using a goniometer (Model 250F1, Rame-Hart, Succasanna, USA). A single water droplet of $10 \mu \mathrm{L}$ was deposited on the dried membrane surface using a gilmount syringe. The reported contact angle was measured from the five various regions on the dried membrane surface. The surface free energy $\left(\Delta \mathrm{G}_{\mathrm{S}_{L}}\right)$ interaction between liquid and membrane surface was calculated using Younge Dupre equation [15].

$-\Delta G_{S}=(1+\operatorname{Cos} \theta) \gamma_{L}^{T}$

where $\theta$ is contact angle value and $\gamma_{L}^{T}$ represent the surface tension of water $\left(72.8 \mathrm{~mJ} / \mathrm{m}^{2}\right)$.

Membrane top surface and cross-section morphology of each sample was studied using SEM (TESCAN, SEM-VEGA 3, Czech Republic). For cross sectional analysis, the membrane samples were initially flashed with liquid nitrogen. Then, membrane samples were sputtered with gold for 
making the surface conductive. The scanning was done at the voltage of $5 \mathrm{kV}$ to ensure proper electron beam focus. The miscibility of GO with PES was evaluated by thermo-gravimetric analysis (TGA) (Model DGT 2000, Perkin Elmer, USA, interfaced to a computer). The analysis was executed from $30{ }^{\circ} \mathrm{C}-900{ }^{\circ} \mathrm{C}$ at the heating rate of $10{ }^{\circ} \mathrm{C} / \mathrm{min}$ under the conditions of nitrogen atmosphere. The XRD patterns of neat PES as well as PES/GO mixed matrix membranes were analyzed using X-ray diffractometer (D8 advance, Bruker, Germany) with monochromatic source of $\mathrm{Cu} k \alpha$ radiation. The samples were studied in $2 \theta$ range with an angle starting from $10^{\circ}$ to $90^{\circ}$ with constant operating voltage of $40 \mathrm{KV}$ and tube current of $30 \mathrm{~mA}$. Tensile strength and elongation ratio for the neat PES and PES/GO membranes were determined using uni-axial mechanical testing machine (Instron, Canton MA). To test the maximum tensile stress, the membrane under test is cut into two dumb bell shaped pieces and measurement corresponds to ASTM D412 standard.

\subsection{Protein adsorption study}

Membrane surface efficacy was studied using bovine serum albumin (BSA) adsorption study. In batch adsorption, BSA $(1.0 \mathrm{~g} / \mathrm{L})$ solution was prepared in $0.1 \mathrm{M}$ phosphate buffer and $\mathrm{pH}$ was varied from 3 to 6 . The membranes were cut into small pieces $(2 \mathrm{~cm} \times 2 \mathrm{~cm})$ and placed in airtight bottles containing BSA solution. Then, the bottles were transferred to a rotating shaker and allowed for BSA adsorption for a brief period of $48 \mathrm{~h}$. The amount of BSA adsorbed on the membrane was measured using UV-Vis spectrophotometer (Shimadzu, Model 275 UV-160A) at a wavelength of $280 \mathrm{~nm}$. BSA adsorption capacity of membrane sample was calculated from the initial concentration of both feed $\left(\mathrm{C}_{\mathrm{f}}\right)$ and final BSA adsorbate $\left(\mathrm{C}_{\mathrm{A}}\right)$ using the following Eq. (2). 
BSA Adsorption $=\left(\frac{C_{f}-C_{A}}{C_{f}}\right) \times 100$

\subsection{Pure water flux, porosity and pore size of the membranes}

Dead end ultrafiltration module (Ultrafiltration cell, Model- S76-400, Spectrum USA) was used for filtration studies of pure water, synthetic melanoidin solution and spent wash effluent respectively. The flux of the membrane $(J)$ was calculated using the Eq. (3).

$$
J=\frac{V}{A \times \mathrm{t}}
$$

where $\mathrm{V}$ is the volume of permeate collected (L), $\mathrm{A}$ is the effective area of the membrane surface $\left(0.0375 \mathrm{~m}^{2}\right), \mathrm{t}$ is the permeation time $(\mathrm{h})$.

The porosity $(\varepsilon)$ of the prepared membranes was determined using gravimetric method and it was determined using the following Eq. (4) [16].

$\varepsilon=\left(\frac{\left(\omega_{1}-\omega_{2}\right)}{\left(\mathrm{d}_{\mathrm{w}} \times P_{v}\right.}\right) \times 100$

where $\omega_{1}$ is the weight of the wet membrane, $\omega_{2}$ is the weight of the dry membrane and $P_{v}$ is the

pore volume of the membrane. Density of the water $\left(\mathrm{d}_{\mathrm{w}}\right)$ and polymer $\left(\mathrm{d}_{\mathrm{p}}\right)$ are $0.998 \mathrm{~g} / \mathrm{cm} 3$ and $1.37 \mathrm{~g} / \mathrm{cm} 3$ respectively. The reported values were calculated from the mean of two experiments. Pore volume of the membrane was calculated the using Eq. (5).

$$
\operatorname{Pv}=\left(\frac{\left(\omega_{1}-\omega_{2}\right)}{\left(d_{w}+\right.}+\frac{\omega_{2}}{d_{p}}\right)
$$


From Eq (4) and (5), the porosity is obtained and shown as Eq. (6).

$\varepsilon=\left(\frac{\left(\omega_{1}-\omega_{2}\right) / d_{w}}{\left(\omega_{1}-\omega_{2}\right) / d_{w}+\left(\omega_{2} / d_{p}\right)}\right)$

The membrane mean pore size was determined using Guerout-Elford-Ferry equation on the basis of pure water flux.

$r_{m}=\sqrt{\frac{(2.9-1.75 \varepsilon) \times 8 \eta l Q}{\varepsilon \times A \times \Delta P}}$

where $\eta$ is the water viscosity $\left(8.9 \times 10^{-4} \mathrm{~Pa} \mathrm{~s}\right), Q$ is the pure water permeation rate $\left(\mathrm{m}^{3} / \mathrm{s}\right), \Delta P$ is the transmembrane pressure $(600 \mathrm{kPa}) A$ is the effective area $\left(\mathrm{m}^{2}\right)$ of the membrane, $l$ is the thickness of the membrane (m).

\subsection{Ultrafiltration performance}

\subsubsection{Preparation of synthetic melanoidin solution}

Synthetic melanoidin solution was prepared following the protocol adopted by Bernardo et al [17]. $4.5 \mathrm{~g}(0.025 \mathrm{M})$ of glucose, $1.88 \mathrm{~g}(0.025 \mathrm{M})$ of glycine and $0.42 \mathrm{~g}(0.005 \mathrm{M})$ of sodium bicarbonate were dissolved in $100 \mathrm{ml}$ of distilled water. Then, the solution was heated overnight at $80^{\circ} \mathrm{C}$ to obtain a concentrated solution.

\subsubsection{Synthetic melanoidin and spent wash ultrafiltration studies}

The obtained model melanoidin solution was used as a feed solution without any pretreatment for filtration runs. Moreover, commercial spent wash effluent was also used in the present study to assess the membrane performance. Commercial spent wash effluent was 
obtained from Trichy Distilleries and Chemicals Ltd., Tiruchirappalli, India. Characteristics of spent wash- analysis are listed in Table 2.

Initially the membranes were compacted at the transmembrane pressure (TMP) of 500 $\mathrm{kPa}$ until steady flux values were obtained. Prior to the experimental runs, initial water flux $\left(\mathrm{A}_{\mathrm{iw}}\right)$ was measured at a TMP of $500 \mathrm{kPa}$. Thereafter, the feed solution was passed by varying the TMP from 100-500 kPa. Flux and samples were measured and collected at a time interval of 10 min. Final water flux $\left(\mathrm{A}_{\mathrm{fw}}\right)$ was again measured to assess the membrane performance. Then, the membranes were cleaned with $0.2 \%$ sodium dodecyl sulphate (SDS) solution at a TMP of 500 $\mathrm{kPa}$ for $0.5 \mathrm{~h}$. Finally, the membrane was again cleaned with double distilled water and flux was monitored. The above similar procedure was followed for the spent wash effluent runs. The spent wash effluent filtration runs were performed at a constant TMP of $400 \mathrm{kPa}$ for $1.5 \mathrm{hr}$.

Flux reduction ratio (FRR) by synthetic melanoidin solution and spent wash effluent on the membranes was calculated using the below Eq. (7).

$\operatorname{FRR}(\%)=\left[\frac{\mathrm{A}_{\mathrm{fw}}}{\mathrm{A}_{\mathrm{iw}}}\right] \times 100$

Color rejection percentage of the synthetic melanoidin and spent wash effluent using membrane filtration was calculated from the absorbance of permeate $\left(\mathrm{A}_{p}\right)$ and feed $\left(\mathrm{A}_{f}\right)$ using Eq. (8). It was quantified at the wavelength of $475 \mathrm{~nm}$ using UV-visible spectrophotometer (Shimadzu, Model 275UV -160A) at characteristic wavelength of $420 \mathrm{~nm}$ [18].

$R=\left(\frac{A_{f}-A_{p}}{A_{f}}\right) \times 100$ 


\section{Results and discussions}

\subsection{Characterization of graphene oxide nanoparticles}

Fig. 1a and 1b show the TEM morphology and XRD pattern of as-prepared GO flakes. TEM analysis shows that GO flakes are homogeneous wrinkled sheets-like multilayer structure. The folded layer ensures the oxidation of graphite to GO [8]. GO multilayer stacks are interconnected by various functional groups. From Fig 1a, TEM images show 1-5 layered graphene oxide sheets with flakes size in the range of 3 to $30 \mu \mathrm{m}$. GO exhibit dense carbonaceous skeleton and thin flakes like structure. The observed few regions of GO sheet also has the dull basal planes. Higher order nanomaterials have significant efficiency in polymer binding and thereby improving the membrane performance [19]. GO nanomaterial possess 2D structure and thus it is expected to intercalate with PES polymer matrix and result in producing a hybrid membrane. Further, X-Ray diffractometer (Model Rigaku Ultima III) equipped with monochromatic $\mathrm{Cu} \mathrm{K} \alpha$ radiation $(\lambda=1.541 \AA)$ was used in determination of $\mathrm{GO}$ crystalline structure and size. The diffraction pattern were collected for $2 \theta$ value ranging from $10^{\circ}$ to $80^{\circ}$ under $40 \mathrm{kV}$. Average particle size (D) of GO was estimated using below Debye Scherrer equation.

$\mathrm{D}=\frac{\mathrm{K} \lambda}{\beta \operatorname{Cos} \theta}$

where $\mathrm{K}$ represents the dimensionless shape factor whose value is $0.9, \lambda$ the $\mathrm{X}$-ray wavelength and $\beta$ the full width at half maximum intensity of peak corresponding to $2 \theta$. XRD analysis display that as-prepared GO flakes (Fig. 1b) are amorphous in nature. The crystallite size was 
found to be $56.21 \mathrm{~nm}$ using Debye Scherrer equation. The small particle sizes are preferred in MMMs preparation due to their better dispersion in polymer matrix and to control the solventnon-solvent exchange rate during phase inversion process [19]. Supporting Fig. 1b shows the FTIR spectra of as-prepared GO particles. The absorption spectral peaks at the wavelength of $3414,1556,1120 \mathrm{~cm}^{-1}$ in $\mathrm{GO}$ particles corresponds to the functional groups of $\mathrm{O}-\mathrm{H}, \mathrm{C}=\mathrm{O}$ and $\mathrm{C}-\mathrm{O}$ respectively. The above functional groups of hydroxyl and carboxyl are expected to increase the hydrophilic property of the membrane surface. These above characteristics may anticipate in aid for the filtration applications.

\subsection{Membrane Characterization}

\subsubsection{Effect of graphene oxide on membrane morphology}

Membrane surface and cross-section morphologies of both neat PES and PES/GO membranes are shown in Fig. 2. As seen in Fig. 2 (G1 to G5), the prepared membranes show the characteristic asymmetric membrane structure of thin skin layer and bottom microporous layer. In membrane surface analysis, smaller pores were noticed in the skin layer of neat PES membranes. PES/GO MMMs exhibited larger pore size and further this was increased for additive incorporated (G4 and G5) PES/GO MMMs. (Fig. 2(a-e)). It is generally well known that formation of membrane structure under phase separation process relies on the both rates of outer diffusion of solvent (DMAc) into a coagulation bath and inner diffusion of non-solvent (water) into a casted thin film [20]. Polymer rich phase formation is initiated by demixing process at the time of immersion of casted film into gelation bath during phase inversion process. The demixing by delayed or instantaneous is favored by the thermodynamic condition between casting solution and non-solvent. The affinity of PES with water is less thus instantaneous 
demixing mechanism is pronounced. While with the addition of hydrophilic materials, the affinity of PES incorporated material leads in delaying the demixing process. As a result From Fig. 2, formation of varied pore size on membrane surface is due to change in the diffusion rate of solvent-non-solvent by the addition of both GO and additives on casting dope solution. Moreover, GO material has binding affinity with PES matrix through both hydrogen bonding and van der Waals force [21]. It is also evident with water flux and pore radius. Fig. 3 shows the water flux compaction data for both neat and modified PES membranes. Compaction data shows the water flux is decreasing and reaches a constant value with respect to time. A high water flux of $57.6 \mathrm{~L} / \mathrm{m}^{2} \mathrm{~h}$ was observed for PES-G0-PAA membrane. The additives are importantly used for formation of large pore size to produce better permeation rate [22]. The additives have a higher affinity to move towards the non-solvent (water) during polymer precipitation. It causes the formation of looser pore matrix. The pore radius of PES-G0-PAA membrane has increased approximately two folds than neat PES membrane. The combination of both additive and nanomaterials further delay the demixing process which is evident is cross sectional analysis. The macrovoid formation reduced in PES/GO MMMs, PES/GO/PAA and PES/GO/LiCl MMMs. The desired ultrafiltration membrane should be of large pore size with lesser macrovoid formation. Hence, the prepared membranes exhibited the preferred morphology. Thus, it is expected to achieve higher flux rate and better performance.

\subsubsection{XRD analysis of graphene oxide nanoparticles and PES/GO mixed matrix membranes}

XRD pattern of pristine PES and PES/GO MMMs are shown in Fig. 4. The characteristic amorphous PES diffraction peak was observed at $2 \theta=18^{\circ}$ on the both pristine and GO modified PES MMMs. A new spectral peak of $13.52^{\circ}$ belonging to GO observed for the GO incorporated 
PES MMMs. This observation clearly signifies that graphene oxide acts as a surface modifying agent, which migrates from the bulk of the casting solution to the membrane surface during the phase inversion process [23]. Moreover, hydrophobic amorphous PES peak intensity diminished for the PES/GO MMMs and remarkably the intensity increased from G2 to G3 MMMs. It is noticed that there is no additional significant diffraction patterns identified on PES/G0-LiCl and PES/G0-PAA MMMs. On the whole, this analysis ensures that the GO intercalated successfully into the PES matrix.

\subsubsection{Thermal behavior of PES/GO mixed matrix membranes}

The TG curves and decomposition temperature value of neat and GO incorporated PES membranes are shown in Fig. 5a and Table 3. Neat PES membrane holds the decomposition temperature of $425{ }^{\circ} \mathrm{C}$. It is interesting to note that decomposition temperature was improved for GO incorporated PES membranes. In case of PES/GO MMMs, PES concentration decreased with the increase of GO concentration (composition, Table 1). Moreover, decomposition temperature of G2 and G3 membrane increased with increase in GO (0.5 and 1.0\%) concentration. The presence of GO in matrix causes delay in the volatilization of MMMs and increase of temperature decomposition for PES/GO MMMs. This ensures that GO has good miscibility in PES matrix [24]. A higher decomposition temperature of $446{ }^{\circ} \mathrm{C}$ was noticed for additive ( $\mathrm{LiCl}$ and PAA) incorporated PES/GO membranes. The minimal difference in temperature decomposition rate for G4 and G5 MMMs were observed. Schematic illustration of interaction between PES and GO-additives are presented in Fig. 5b. $\mathbf{~ L i C l}$ has stronger affinity with GO through metal oxygen interactions. In case of PAA, polar-polar interactions are held together with GO. Besides, PES constitutes electron donating sulfone group and it result in 
stronger binding with $\mathrm{GO}$ through the available free functional group of $-\mathrm{OH}$ and $-\mathrm{COOH}$. Additive interaction has significant influence on alteration of membrane physical properties [25]. As a result, temperature decomposition study shows MMMs miscible property was varied with addition of additives and increase in GO concentration. It is mainly due to interaction between the synergetic effect of both additives and GO complex. The above structural affinity has interlinkage with GO functional atoms and result in producing hybrid nanocomposite membranes.

\subsubsection{Mechanical properties of PES/GO mixed matrix membranes}

Table 3 shows the tensile stress and elongation percentage of pristine PES and GO incorporated PES membranes. The tensile stress and elongation ration of pristine PES membrane are $0.99 \mathrm{MPa}$ and 7.4 respectively. The tensile stress reached maximum up to 2.81 and $2.94 \mathrm{MPa}$ for PES/GO-LiCl membrane. Moreover, tensile stress increased with increase of addition of GO from $0.5(\mathrm{G} 2)$ to $1.0 \%(\mathrm{G} 3)$ MMMs. It reveals that robust GO particle entangled effectively with PES matrix. Hence, modified MMMs can also operate under optimum transmembrane pressure to obtain higher flux rate. It is remarkable to understand that elongation ratio decreased for GO incorporated PES MMMs. This might be due to the enhancement of surface porosity and decrease of polymer concentration of the corresponding PES/GO MMMs [26]. From the supplementary Table 1, higher porosity of $81 \%$ noticed for PES/G0-PAA MMMs. Thus, elongation ratio decreased lowered up to 5.02 for PES/G0-PAA MMMs. On the whole of membrane characterization studies, GO and GO-additives has good miscibility with PES matrix.

\subsection{BSA adsorption study}

Surface functionality of neat PES and modified PES MMMs was studied by employing static BSA adsorption test. Fig. 6. shows the BSA adsorption with respect to pH on prepared 
membranes. It is generally well known that adsorption is a surface phenomenon. The BSA adsorption capacity of membranes increases with increase of $\mathrm{pH}$ from 3 to 5 . It is due to the surface charge of the BSA molecules and the isoelectric point of BSA is 4.7. At the isoelectric point, BSA attains a neutral charge and it results in higher adsorption capacity of the membranes at $\mathrm{pH} 5$ [27]. However, at $\mathrm{pH}$ of above 5, BSA acquire negative charges and it causes lesser binding interactions with membranes. In this test, PES/GO, PES/GO-PAA and PES/GO-LiCl MMMs showed lesser BSA adsorption on the membrane surface. It is due to the increase in surface free energy of the membrane surface (Table 1) [27]. It is very important to note that neat PES which exhibited a higher contact angle value of $70.8^{\circ}$ and it was reduced to $58.4^{\circ}$ for the modified PES/GO-PAA MMMs. GO possesses various functional molecules of hydroxyl, carboxyl, carbonyl and epoxy groups [8]. The existence of these inherent groups leads in improvement of hydrophilicity on the PES mixed matrix membrane surface. Thus, the protein adsorption got decreased at higher $\mathrm{pH}$. Thus, this experiment shows that membrane surface functionality was improved for MMMs. These properties aid in the treatment of distillery effluents.

\subsection{Mixed matrix membranes performance in the treatment of synthetic melanoidin and} distillery spent wash effluent

Melanoidins are produced from the carbonyl groups of reducing sugars and the amino groups of amino acids, peptides or proteins under higher temperature [3]. Literature shows that melanoidin molecular weights are in the range from 5 to14 $\mathrm{kDa}$ [28]. The main cause of color arises is due to the presence of mealanoidin. The filtration analysis of both synthetic melanoidin and distillery spent wash effluent are discussed as follows. Fig. 7. shows the synthetic 
melanoidin solution flux of membranes with respect to different transmembrane pressure (TMP). It also clearly elucidates that flux values deviates from the non-linearity with respect to TMP. This phenomenon is due to the either adsorption or accumulation of solute particles on the membrane surface [5]. The similar pattern is observed on all the fabricated membranes and the flux was increased up to the TMP of $400 \mathrm{kPa}$. Thus, the TMP was maintained for the spent wash effluent filtration under constant TMP. Spent wash effluent flux was decreasing with increase of

time (Fig. 8). As compared to both effluents, spent wash effluent flux was lower for all the membranes. The lower permeate flux was noticed for distillery spent wash effluent while compared to synthetic melanoidin solution. It is due to higher flux reduction ratio (Fig. 9) and also based on the feed characteristics (Table 2). Spent wash constitutes higher amount of COD which arises from various pollutants from various unit operations of distillery industry $[1,3]$. On the other hand, PES/GO MMMs showed better flux performance than neat PES membrane (Fig. 7-8). The improvement in flux property is ascertained due to improvement of hydrophilic property on the membrane surface. It is also evident in flux recovery ratio analysis (Fig. 9). Higher flux reduction ratio was observed for the neat PES membrane. It is important to note that FRR was progressively improved for PES/GO MMMs and additive incorporated PES/GO-PAA as well as PES/GO-LiCl membrane. This study explicitly indicates that effluent filtration run has higher efficiency under low TMP.

\subsection{Color rejection by PES/GO MMM's}

Fig. 10. shows the color removal efficiency of the membranes towards synthetic melanoidin solution and spent wash effluents respectively. A higher color removal of 59\% and 54\% was obtained in G3 (PES/GO1\%) membrane for synthetic melanoidin solution and spent 
wash effluent respectively. The color removal was slightly reduced for G4 (PES/GO-LiCl) and G5 (PES/GO- PAA) MMMs. It is due to the increase of pore radius for additive incorporated PES/GO MMMs [29]. Thus, they allow solutes to pass through permeate side of the membrane. Nataraj at al. [30] observed up to $99 \%$ COD removal for the distillery effluent using reverse osmosis membranes. Supplementary Table 2. shows the comparison of distillery effluent treatment of various membrane operations [31]. However, in the present study, modified PES/GO MMMs were effectively removed a significant amount of color at a nominal flux rate for distillery effluent under low transmembrane pressure. On the whole, this studies shows that additive combined PES/GO membranes had a lesser flux reduction ratio with better flux performance. The prepared membrane would be an effective material for preliminary treatment and followed for the water reclamation process.

\section{Conclusions}

In this study, nanocomposite PES/GO membranes and influence of different additives such as PAA and $\mathrm{LiCl}$ with PES/GO nanocomposite membranes on the structure and performance of the membrane was also examined. The characterization of GO flakes using TEM and XRD analysis show multilayer structure and smaller crystalline size of $56.21 \mathrm{~nm}$. Moreover, GO has good miscibility with PES membrane and it is evident in TGA, mechanical strength and XRD analysis. The functional groups of GO has better interlinked with additives and PES polymer. PES/GO-PAA and PES/GO-LiCl MMMs holds larger pore size which is evident from the SEM images. It was found that hydrophilicity, pure water flux was improved for PES/GO membrane and further improved remarkably up to PES/GO/PAA membrane (Contact angle value- $58.20^{\circ}$ and water flux $57 \mathrm{~L} / \mathrm{m}^{2} \mathrm{~h}$ ). Further, Static adsorption study with BSA solution 
revealed that PES/GO/PAA combination exhibited lower fouling, showing better antifouling ability. These studies elucidates that modified MMMs surface functionality was enhanced. Filtration performance studies show that synthetic melanoidin solution exhibited higher flux and better color rejection while compared to spent wash effluents. Flux studies shows modified MMMs can operate under lower TMP. From the above studies, it is inferred that additive combined PES/GO membrane has better characteristic membrane properties and lesser flux reduction ratio for both synthetic melanoidin solution and spent wash effluents. Overall, this study indicates that utilization of as as-prepared hydrophilic PES/GO MMMs can be used to reduce the organic load in distillery effluent. These studies also give insight to other researchers for the use of nanomaterials in membrane fabrication for their improved performance in distillery effluent application.

\section{Acknowledgement}

The authors thank Dr. S. Ramaprabhu, Department of Physics, Indian Institute of Technology, Madras, for his guidance and support in accomplishing synthesis of graphene oxide (GO). The authors also thank Mr. K. Sankaran, Prime Minister Fellow, Department of Energy and Environment, National Institute of Technology, Tiruchirappalli for detailing the characteristics of spent wash distillery wastewater and helping for work start-up. 


\section{References}

[1] K. Sankaran, M. Premalatha, M. Vijayasekaran, V.T. Somasundaram, DEPHY project: Distillery wastewater treatment through anaerobic digestion and phycoremediation - A green industrial approach, Renew. Sustain. Energy Rev. 37 (2014) 634-643.

[2] R.N Bharagava, R. Chandra, Effect of bacteria treated and untreated post methanated distillery effluent (PMDE) on seed germination, seedling growth and amylase activity in Phaseolus mungo L., J. Hazard. Mater. 180 (2010) 730-734.

[3] Y. Satyawali, M. Balakrishanan, Treatment of distillery effluent in a membrane bioreactor (MBR) equipped with mesh filter, Sep. Purif. Technol. 63 (2008) 278-286.

[4] D. Pant, A. Adholeya, Biological approaches for treatment of distillery wastewater: A review, Bioresour. Technol. 98 (2007) 2321-2334.

[5] M. Cheryan, Ultrafiltration and Microfiltration Handbook, Technomic, Lancaster, PA, 1998.

[6] H. Susanto, M. Ulbticht, Characteristics, performance and stability of Polyethersulfone ultrafiltration membranes prepared by phase separation method using different macromolecular additives, J. Membr. Sci. 327 (2009) 125-135.

[7] J. Kim, B. Van der Bruggen, The use of nanoparticles in polymeric and ceramic membrane structures, Review of manufacturing procedures and performance improvement for water treatment, Environ. Pollut. 2010, 158, 2335-2349. 
[8] Y. Zhu, S. Murali, W. Cai, X. Li, J.W. Suk, J.R. Potts, R.S. Ruoff, Graphene and Graphene Oxide: Synthesis, Properties, and Applications, Adv. Mater. 22 (2010) 39063924.

[9] J. Lee, H. R Chae, Y. J. Won, K. Lee, C. H. Lee, H. H. Lee, J. M. Lee, Graphene oxide nanoplatelets composite membrane with hydrophilic and antifouling properties for wastewater treatment, J. Membr. Sci. 448 (2013) 223-230.

[10] S. Zinadini, A.A. Zinatizadeh, M. Rahimi, V. Vatanpour, H. Zangeneh, Preparation of a novel antifouling mixed matrix PES membrane by embedding graphene oxide nanoplates, J. Membr. Sci. 453 (2014) 292-301.

[11] J. Zhang, Z. Xu, M. Shan, B. Zhou, Y. Li, B. Li, X. Qian,. Synergetic effects of oxidized carbon nanotubes and graphene oxide on fouling control and anti-fouling mechanism of polyvinylidene fluoride ultrafiltration membranes, J. Membr. Sci. 448 (2013) 81-92.

[12] A.L. Ahmad, A.A. Abdulkarim, B.S. Ooi, S. Ismail, Recent development in additives modifications of polyethersulfone membrane for flux enhancement, Chem. Eng. J. 223 (2013) 246-267.

[13] A. Kaniyoor, T.T. Baby, S. Ramaprabhu, Graphene synthesis via hydrogen induced low temperature exfoliation of graphite oxide, J. Mater. Chem. 20 (2010) 8467-8469.

[14] Y. Lee, S. Kim, H.-i. Lee, H. Jeong, A. Raghu, K. Reddy, B. Kim, Graphite oxides effective fire retardants of epoxy resin, Macromol. Res. 19 (2011) 66-71. 
[15] D.Y. Kwok, A.W. Neumann, Contact angle interpretation in terms of solid surface tension, Colloid Surf. A. 161 (2000) 31-48.

[16] N. A. A. Hamid, A.F. Ismail, T. Matsuura, A.W. Zularisam, W.J. Lau, E. Yuliwati, M.S. Abdullah, Morphological and separation performance study of polysulfone/titanium dioxide (PSF/TiO2) ultrafiltration membranes for humic acid removal. Desalination. 273 (2011) 85-92.

[17] E.C. Bernardo, R. Egashira, J. Kawasaki, Decolorization of molasses wastewater using activated carbon prepared from cane bagasse, Carbon 35 (1997) 1217-1221.

[18] C. Raghukumar, G.Rivonkar, Decolorization of molasses spent wash by the white rot fungus Flavodon flavus, isolated from marine habitat. Appl. Microbiol. Biotechnol. 55 (2001) 510-514.

[19] P. Wang, J. Ma, F. Shi, Y. Ma, Z. Wang, X. Zhao, Behaviors and Effects of Differing Dimensional Nanomaterials in Water Filtration Membranes through the Classical Phase Inversion Process : A Review, Ind. Eng. Chem. Res. 52 (2013) 10355-10363.

[20] M. Safarpour, A. Khataee, V. Vatanpour, Effect of reduced graphene oxide/ $/ \mathrm{TiO}_{2}$ nanocomposite with different molar ratios on the performance of PVDF ultrafiltration membranes, Sep. Purif. Technol. 140 (2015) 32-42.

[21] Z. Xu, J. Zhang, M. Shan, Y. Li, B. Li, J. Niu, B. Zhou, X. Qian, Organosilanefunctionalized graphene oxide for enhanced antifouling and mechanical properties of polyvinylidene fluoride ultrafiltration membranes, J. Membr. Sci. 458 (2014) 1-13. 
[22] B.M. Ganesh, A.M. Isloor, A.F. Ismail, Enhanced hydrophilicity and salt rejection study of graphene oxide-polysulfone mixed matrix membrane, Desalination 313 (2013) 199207.

[23] X. Chenxi, C. Yuancheng, K. Ravi, W. Xu, W. Xu, and S. Keith, A polybenzimidazole/sulfonated graphite oxide composite membrane for high temperature polymer electrolyte membrane fuel cells, J. Mater. Chem. 21 (2011) 11359-11364.

[24] J. Dasgupta, S. Chakraborty, J. Sikder, R. Kumar, D. Pal, S. Curcio, E. Drioli, The effects of thermally stable titanium silicon oxide nanoparticles on structure and performance of cellulose acetate ultrafiltration membranes, Sep. Purif. Technol. 133 (2014) 55-68.

[25] G. Arthanareeswaran, T.K. Sriyamuna Devi, D. Mohan, Development, characterization and separation performance of organic-inorganic membranes. Part II. Effect of additives, Sep. Purif. Technol. 67 (2009) 271-281.

[26] G.D. Vilakati, E.M.V. Hoek, B.B. Mamba, Probing the mechanical and thermal properties of polysulfone membranes modified with synthetic and natural polymer additives, Polymer Testing, 34, (2014). 202-210.

[27] K. Narasaiah, G.P. Aggarwal, Transmission analysis in ultrafiltration of ternary protein mixture through a hydrophilic membrane, J. Membr. Sci. 287 (2007) 9-18.

[28] H.Y. Wang, H. Qian, W.R. Yao, Melanoidins produced by the Maillard reaction: Structure and biological activity, Food Chem. 128 (2011) 573-584. 
[29] M. Kumar, M. Ulbricht, Novel ultrafiltration membranes with adjustable charge density based on sulfonated poly(arylene ether sulfone) block copolymers and their tunable protein separation performance, Polymer 55 (2014) 354-365.

[30] S.K. Nataraj, K.M. Hosamani, T.M. Aminabhavi, Distillery wastewater treatment by the membrane-based nanofiltration and reverse osmosis processes, Water Res. 40 (2006) $2349-2356$.

[31] U.K. Rai, M. Muthukrishnan, B.K. Guha, Tertiary treatment of distillery wastewater by nanofiltration, Desalination 230 (2008) 70-78. 


\section{Table Captions}

Table 1. Composition of casting solution for fabricated membranes and determination of hydrophilicity as well as mean pore radius

Table 2. Characteristics of spent wash

Table 3. Mechanical and thermal properties of neat PES and PES/GO membranes. 


\section{Figure Caption}

Fig. 1a. TEM image of Graphene oxide nanoparticles

Fig. 1b XRD pattern of Graphene oxide nanoparticles

Fig. 2. Top and cross-sectional SEM images of the prepared (G1) Neat PES, (G2) $0.5 \%$ GO/PES, (G3) 1\% GO/PES, (G4) PES/GO-LiCl, (G5) PES/GO-PAA.

Fig. 3. Water flux compaction data of neat PES/GO and modified PES/GO MMMs

Fig. 4. XRD analysis of neat PES membrane and PES/GO MMMs.

Fig. 5a. Thermogravimetric analysis for PES/GO MMMs.

Fig. 5b. Schematic representation of PES-GO-additive interactions

Fig. 6. Effect of $\mathrm{pH}$ on static BSA adsorption of PES/GO MMMs.

Fig. 7. Effect of transmembrane pressure (TMP) on permeate flux of synthetic melanoidins.

Fig. 8. Spent wash effluent Permeate flux profile of PES/GO MMMs at a TMP of $400 \mathrm{kPa}$.

Fig. 9. Effect of PES/GO MMMs performance on flux recovery ratio of synthetic melanoidin and spent wash effluent.

Fig. 10. Effect of PES/GO MMMs performance on color removal of both synthetic melanoidin and spent wash effluent solution. 


\section{Table 1}

Composition of casting solution for fabricated membranes and determination of hydrophilicity as well as mean pore radius

\begin{tabular}{|c|c|c|c|c|c|c|c|}
\hline \multirow[t]{2}{*}{$\begin{array}{l}\text { Membrane } \\
\text { Code }\end{array}$} & \multicolumn{4}{|c|}{$\begin{array}{l}\text { Polymer and additive composition } \\
\text { (17.5\% Polymer }) /(82.5 \% \text { DMAc })\end{array}$} & \multirow[t]{2}{*}{$\begin{array}{l}\text { Contact } \\
\text { angle }\end{array}$} & \multirow{2}{*}{$\begin{array}{l}\text { Surface } \\
\text { free } \\
\text { energy } \\
\left(\mathrm{mJ} \mathrm{m}^{-2}\right)\end{array}$} & \multirow[t]{2}{*}{$\mathrm{r}_{\mathrm{m}}(\mathrm{nm})$} \\
\hline & PES & GO & PAA & $\mathrm{LiCl}$ & & & \\
\hline G1 & 100 & 0 & 0 & 0 & 70.8 & 96.74 & 2.09 \\
\hline $\mathrm{G} 2$ & 99.5 & 0.5 & 0 & 0 & 64.5 & 104.14 & 2.76 \\
\hline G3 & 99 & 1 & 0 & 0 & 61.2 & 107.87 & 3.18 \\
\hline G4 & 98 & 1 & 0 & 1 & 60.8 & 108.31 & 3.74 \\
\hline G5 & 98 & 1 & 1 & 0 & 58.4 & 110.94 & 4.08 \\
\hline
\end{tabular}

\section{Table 2}

Characteristics of spent wash

\begin{tabular}{cc}
\hline Parameter & Concentration range \\
Color & Dark brown \\
Temperature & $37-43^{\circ} \mathrm{C}$ \\
Conductivity & $32-37 \mathrm{mS} / \mathrm{cm}$ \\
Turbidity (NTU) & 40 \\
TDS & $35,200-45,200 \mathrm{ppm}$ \\
COD & $25,000-40,000 \mathrm{ppm}$ \\
\hline
\end{tabular}




\section{Table 3}

Mechanical and thermal properties of neat PES and PES/GO membranes.

\begin{tabular}{llll}
\hline $\begin{array}{l}\text { Membrane } \\
\text { code }\end{array}$ & $\begin{array}{l}\text { Tensile stress } \\
\text { at break } \\
(\mathrm{MPa})\end{array}$ & $\begin{array}{l}\text { Elongation break } \\
(\mathrm{mm})\end{array}$ & $\begin{array}{l}\text { Decomposition } \\
\text { Temperature } \\
\left(\mathrm{T}_{\mathrm{d}}{ }^{\circ} \mathrm{C}\right)\end{array}$ \\
\hline $\mathrm{G} 1$ & $0.99(0.37)$ & $7.4(1.9)$ & 425 \\
$\mathrm{G} 2$ & $1.63(0.64)$ & $6.9(2.7)$ & 433 \\
$\mathrm{G} 3$ & $2.73(0.84)$ & $6.1(2.4)$ & 439 \\
G4 & $2.81(0.52)$ & $5.9(1.8)$ & 446 \\
G5 & $2.94(0.90)$ & $5.2(1.1)$ & 448 \\
\hline
\end{tabular}




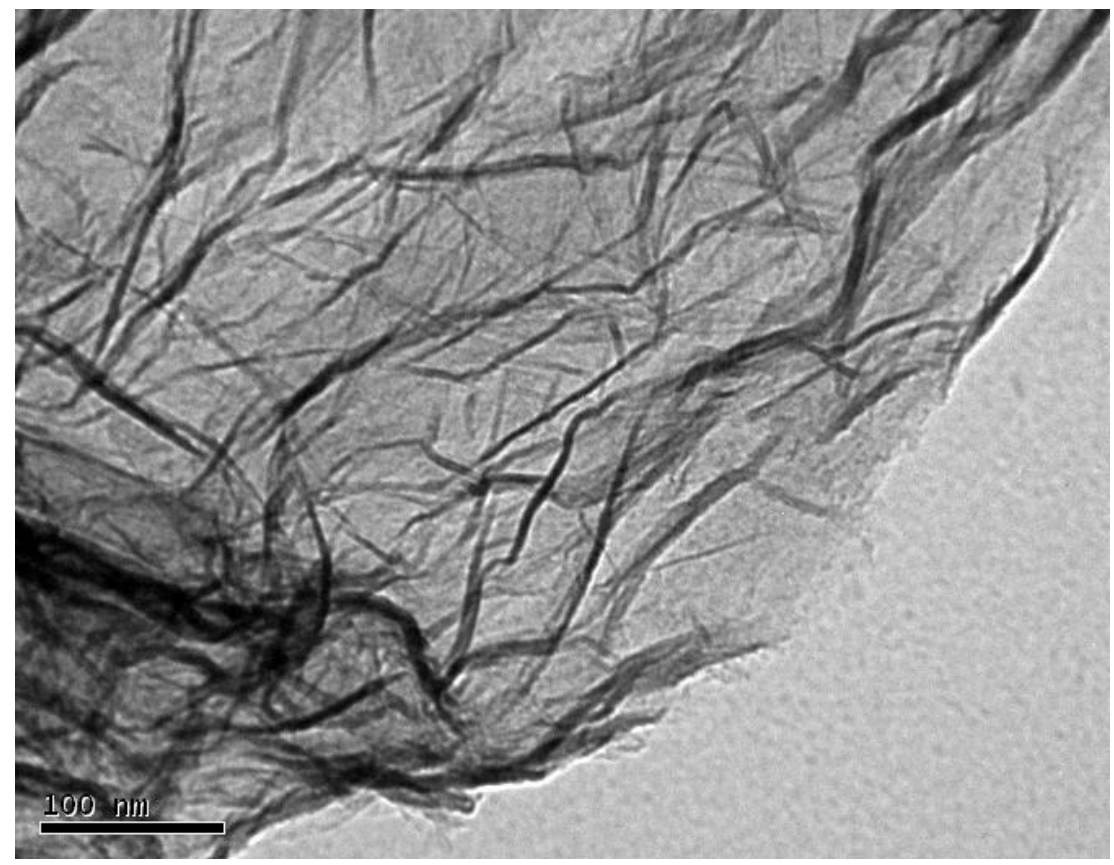

Fig. 1a. TEM image of graphene oxide flakes

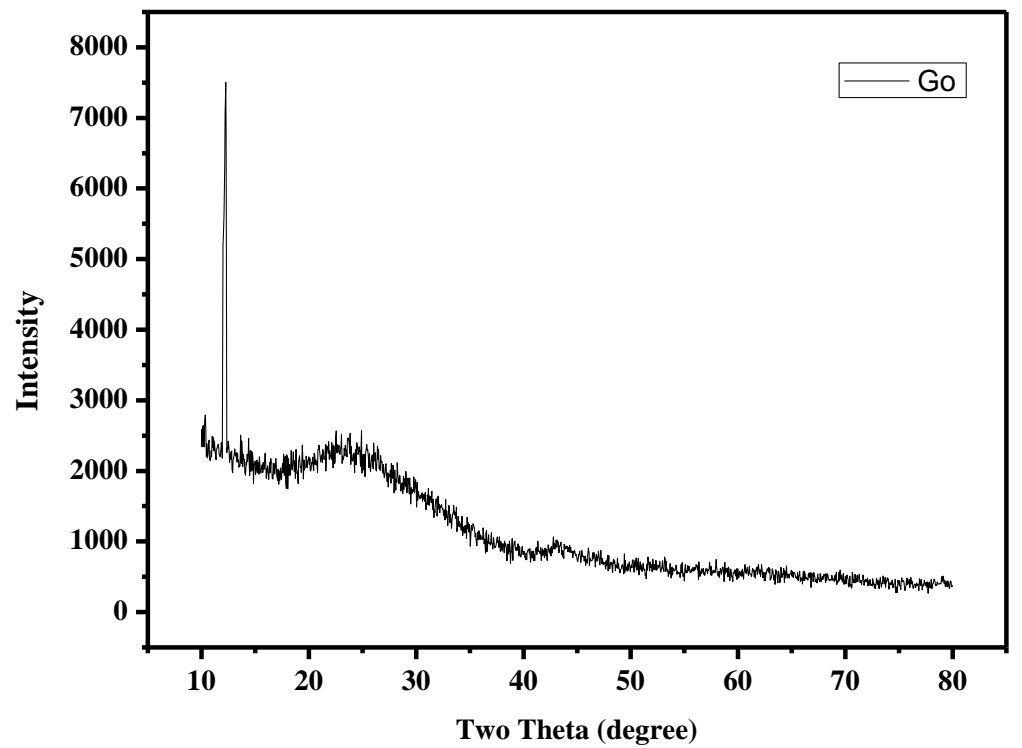

Fig. 1b XRD pattern of Graphene oxide flakes 

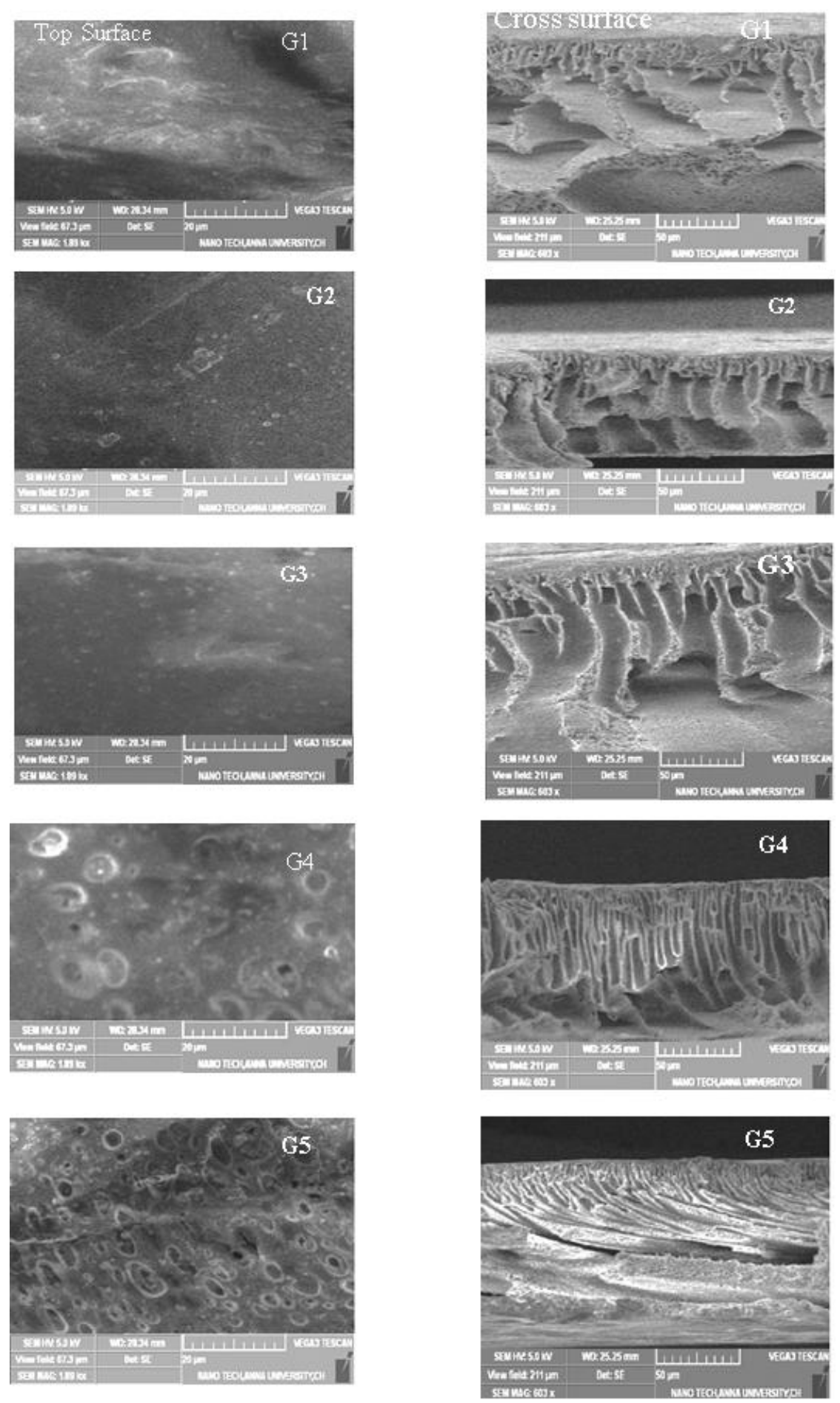

Fig. 2. Top and cross-sectional SEM images of the prepared (G1) Neat PES, (G2) $0.5 \%$ GO/PES, (G3) 1\% GO/PES, (G4) PES/GO-LiCl, (G5) PES/GO-PAA. 


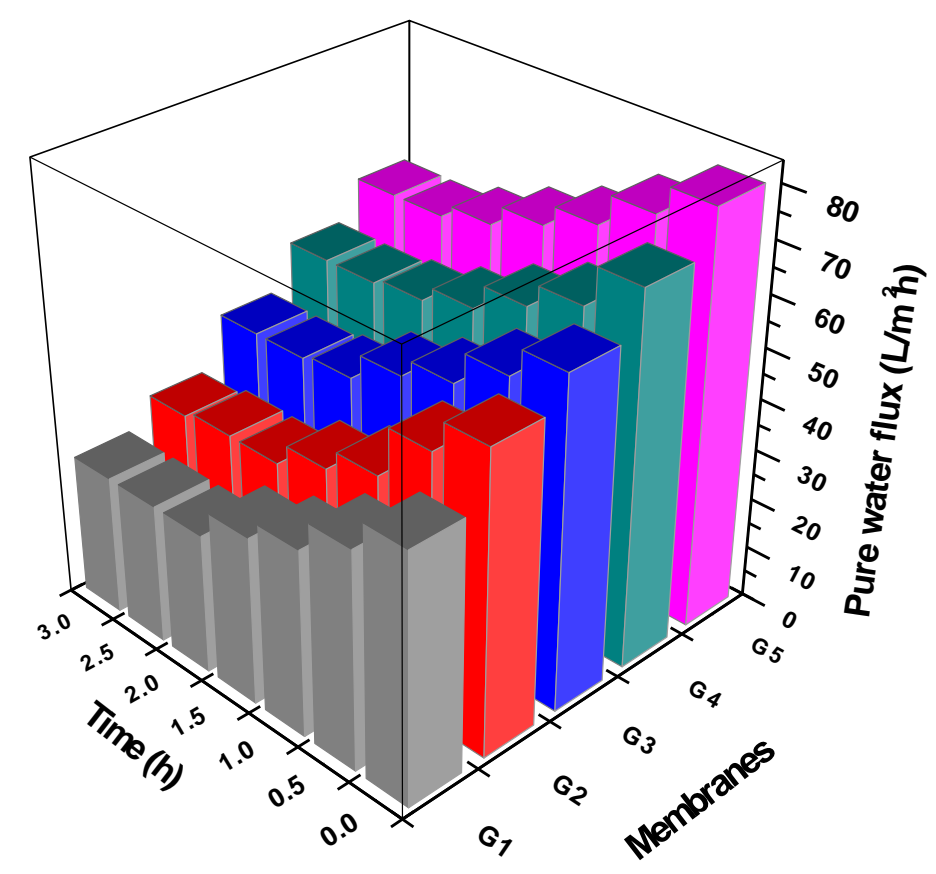

Fig. 3. Water flux compaction data of neat PES/GO and modified PES/GO MMMs. 


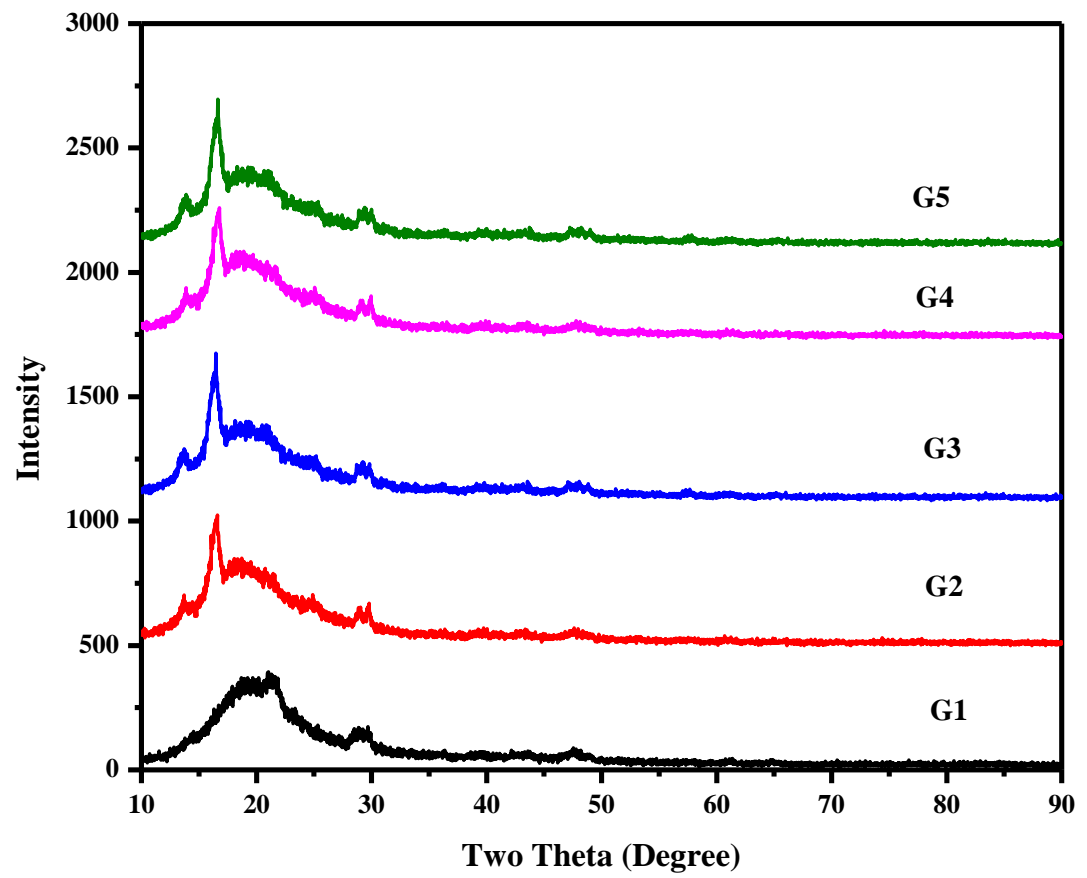

Fig. 4. XRD analysis of neat PES membrane and PES/GO MMMs. 


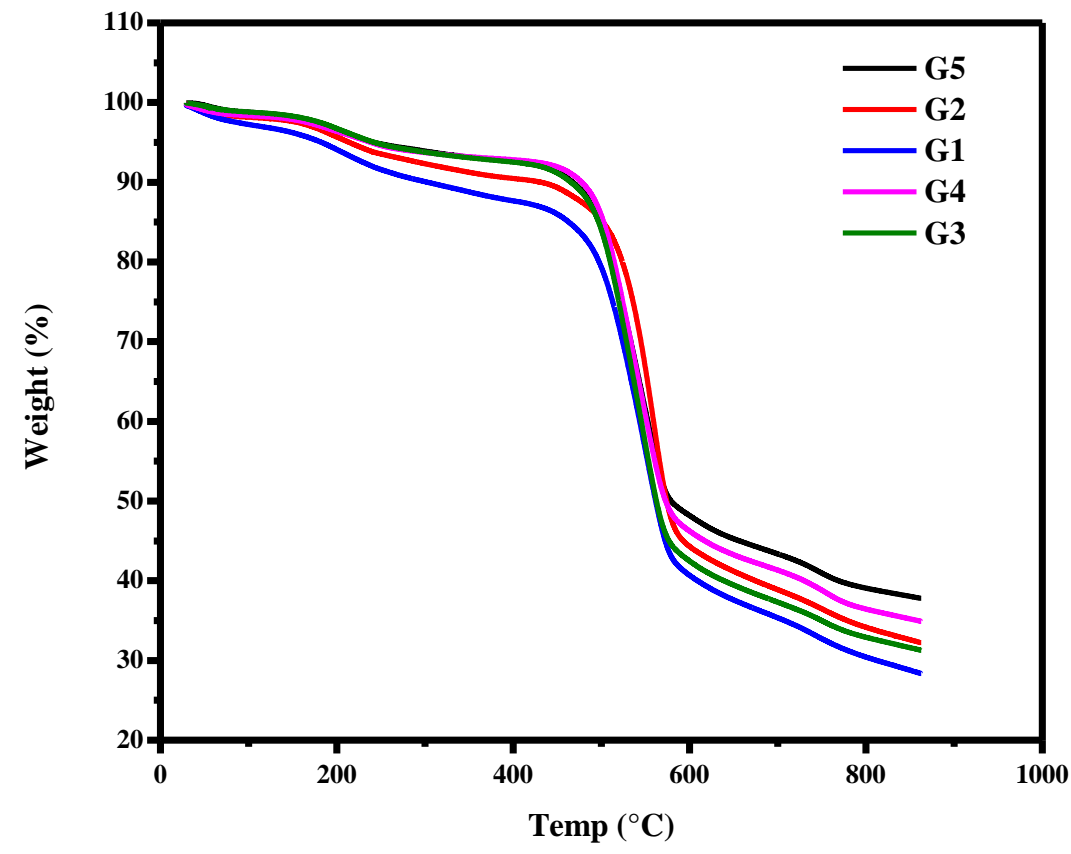

Fig. 5a. Thermogravimetric analysis of neat PES and PES/GO MMMs. 

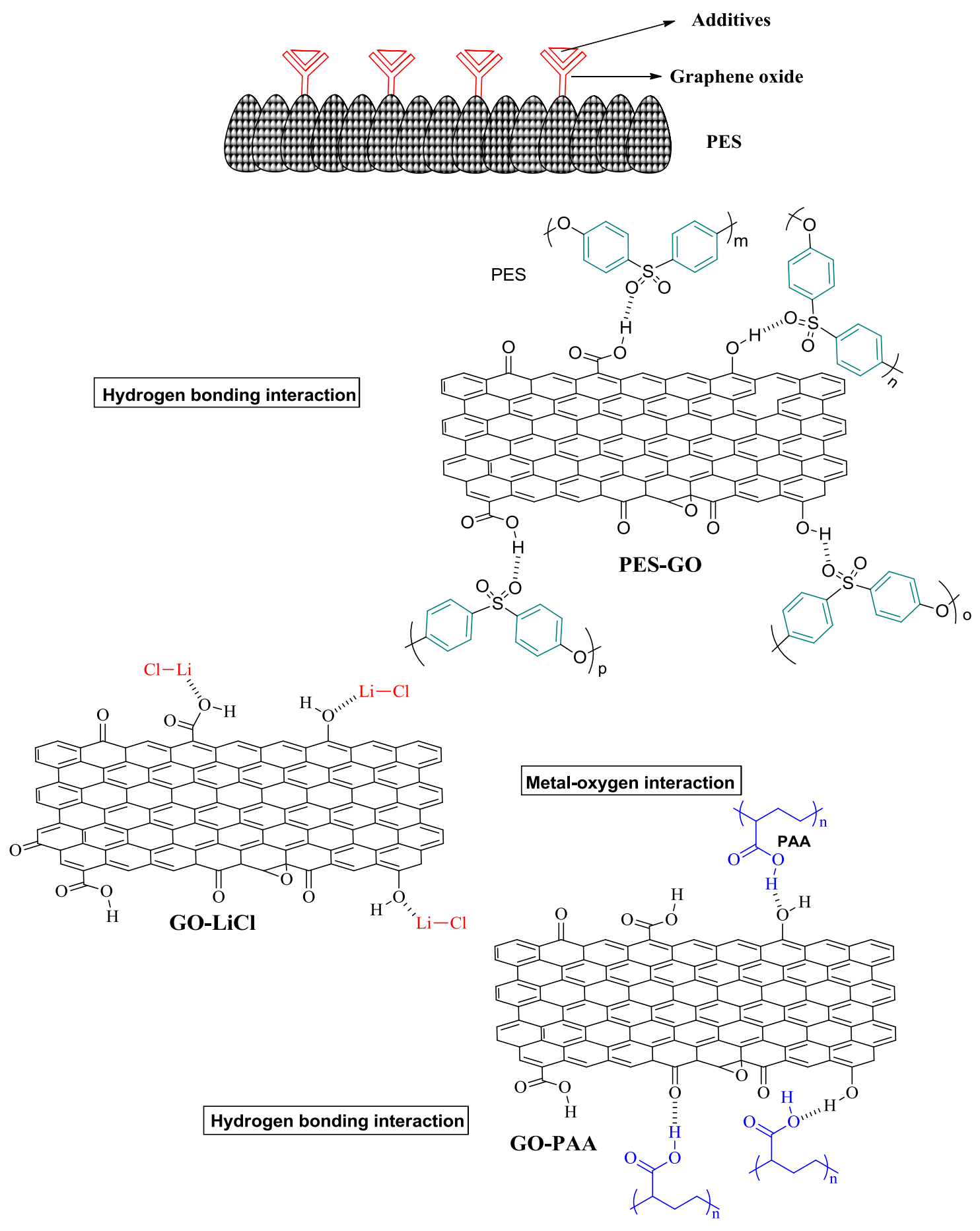

Fig. 5b. Schematic representation of PES-GO-additive interactions. 


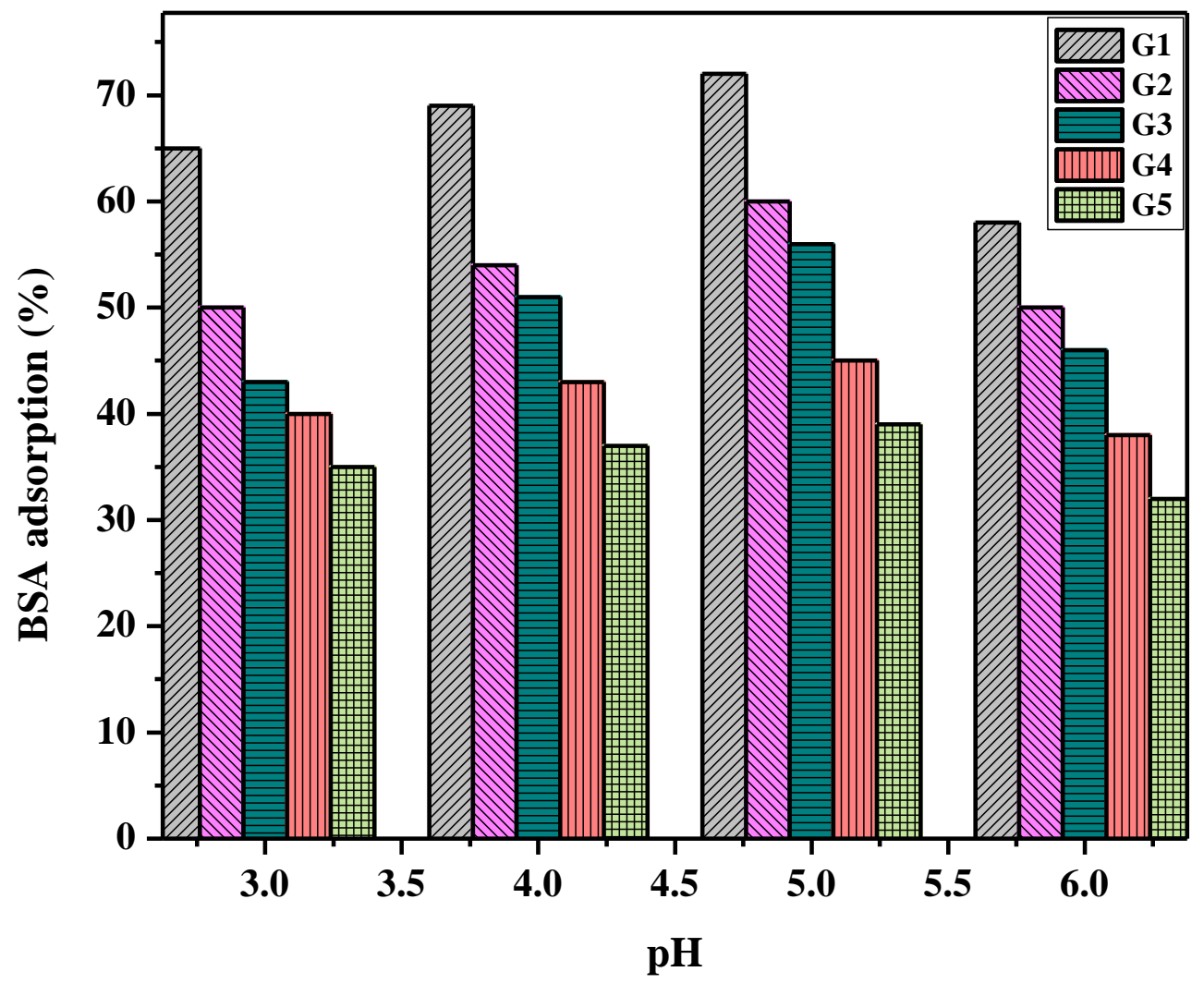

Fig. 6. Effect of $\mathrm{pH}$ on static BSA adsorption of PES/GO MMMs. 


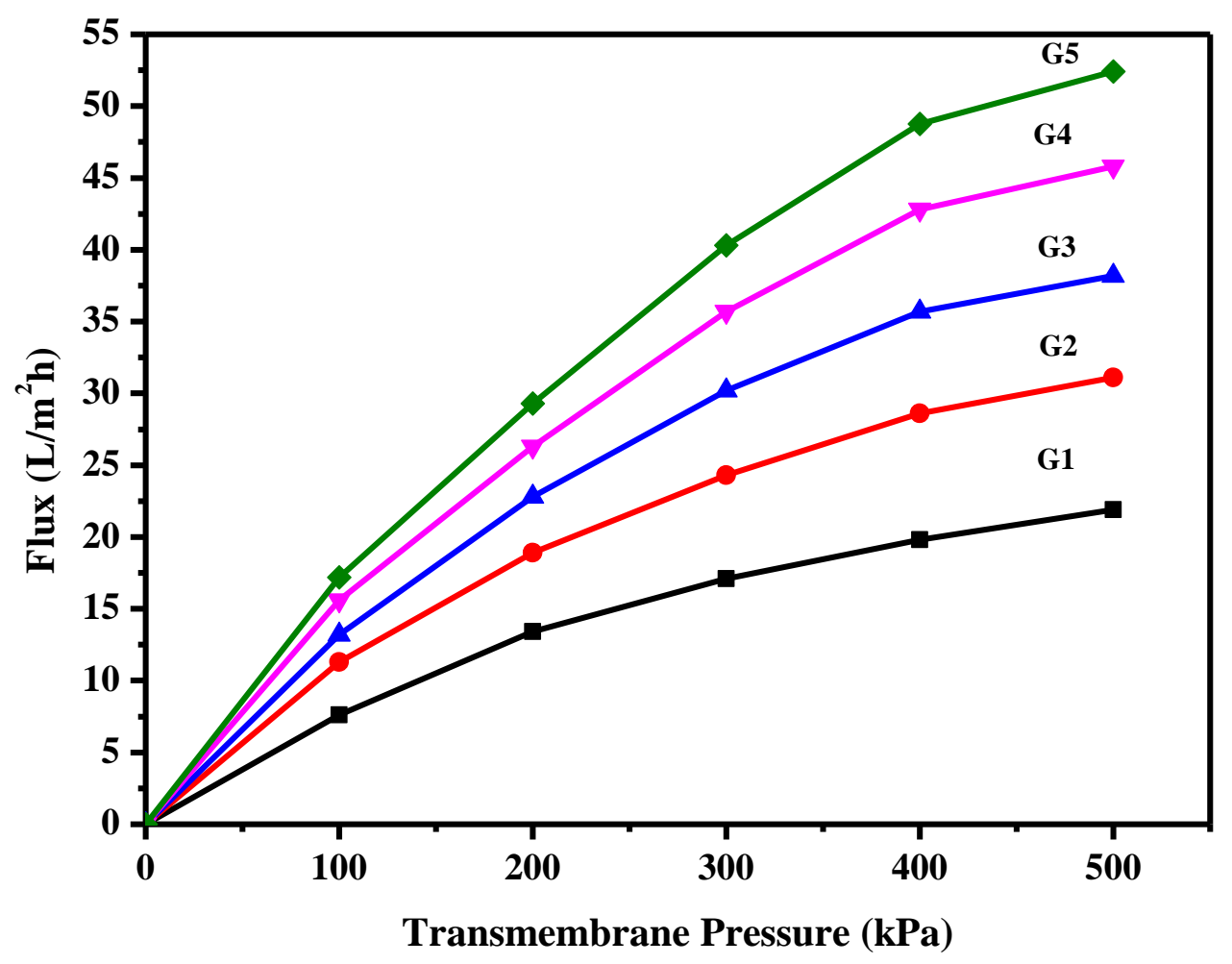

Fig. 7. Effect of transmembrane pressure (TMP) on permeate flux of synthetic melanoidins. 


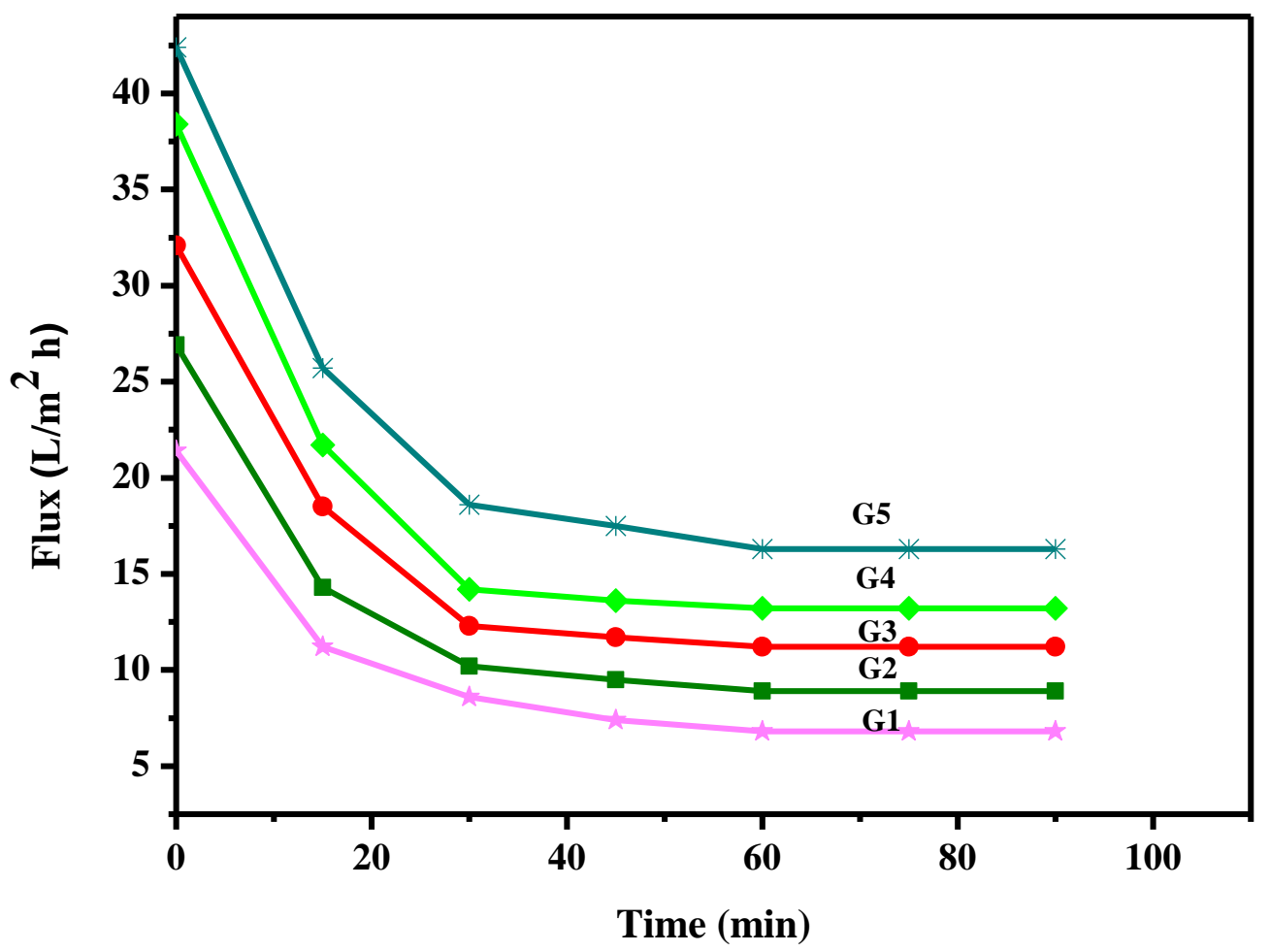

Fig. 8. Spent wash effluent Permeate flux profile of PES/GO MMMs at a TMP of $400 \mathrm{kPa}$. 


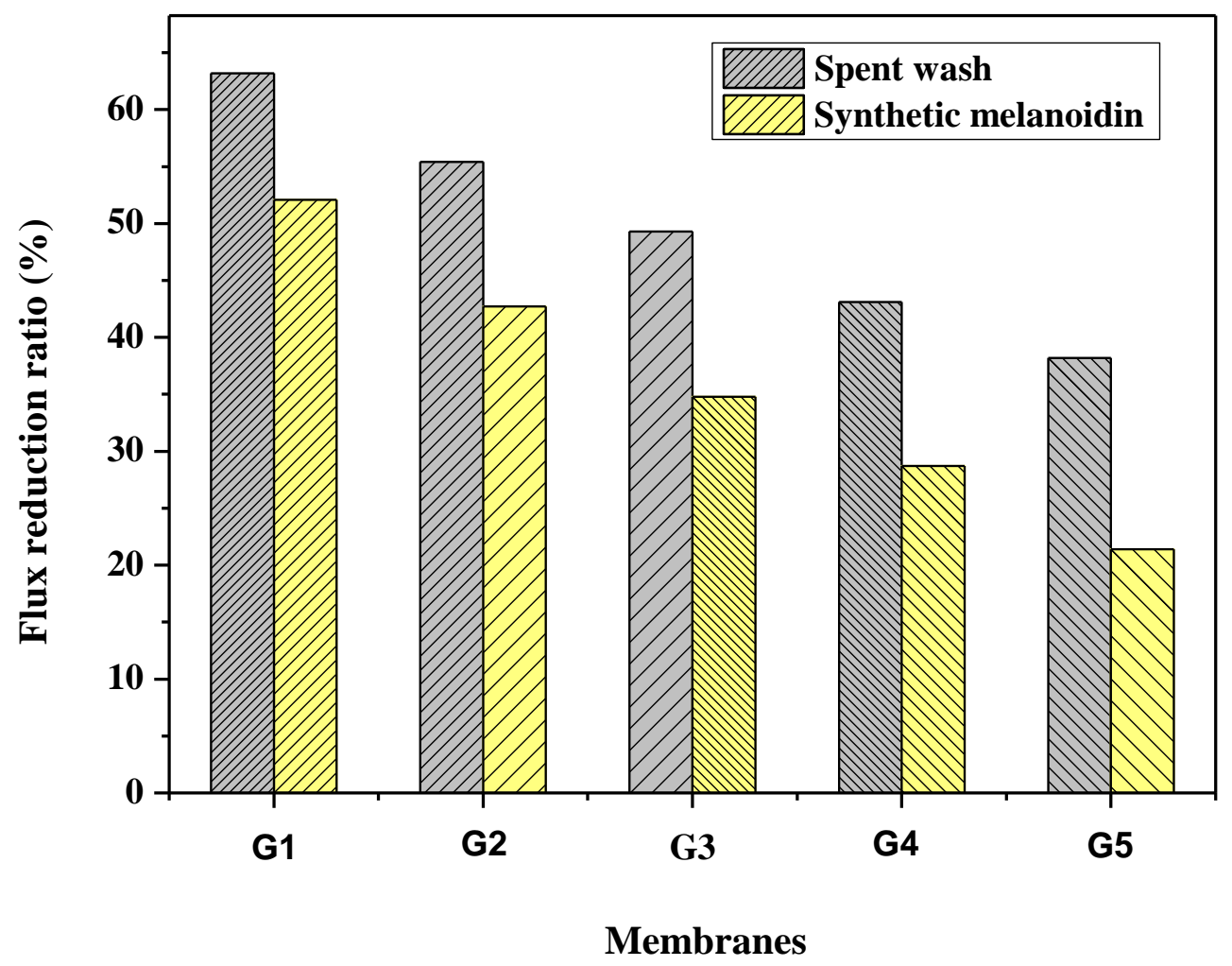

Fig. 9. Effect of PES/GO MMMs performance on flux recovery ratio of synthetic melanoidin and spent wash effluent. 


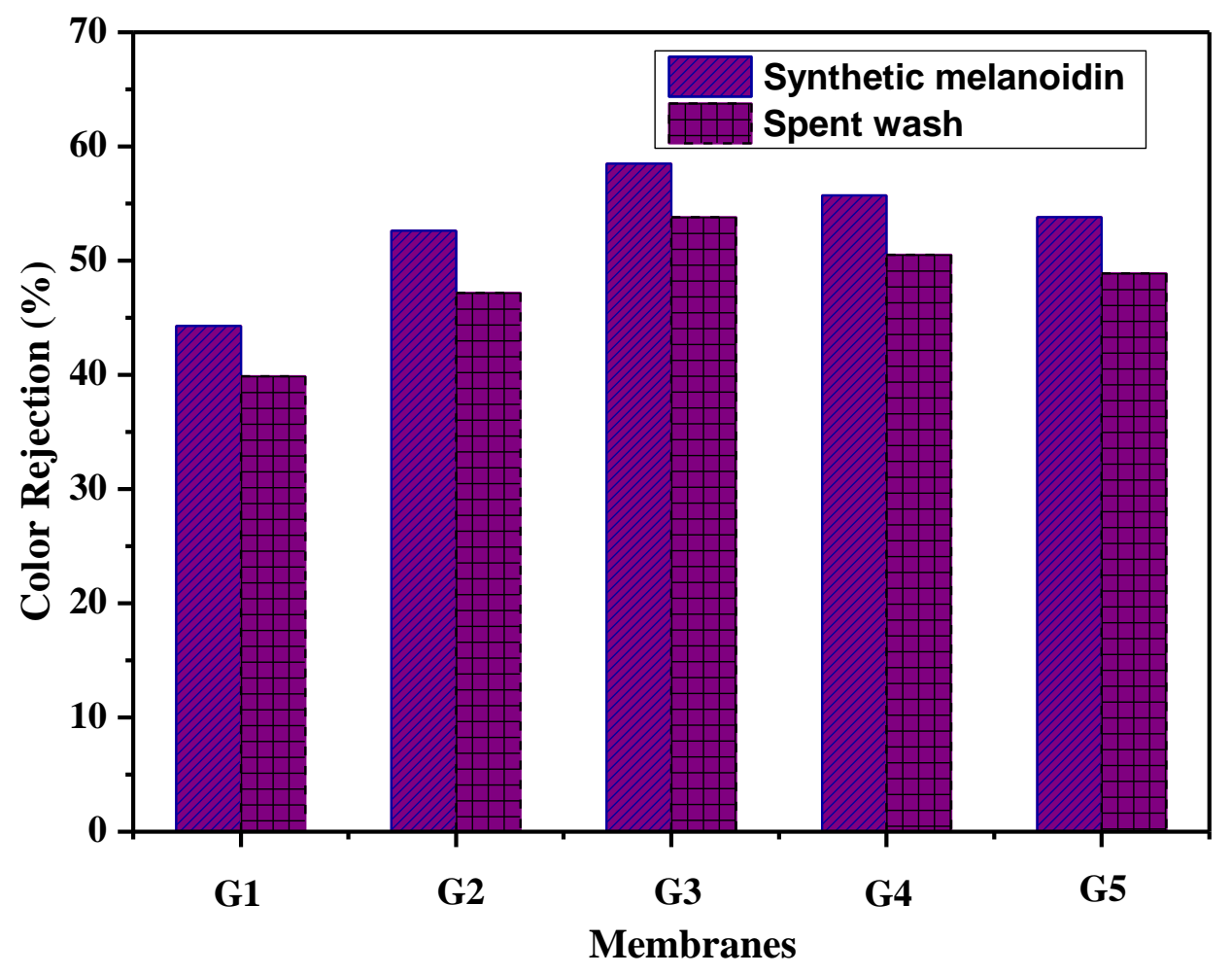

Fig. 10. Effect of PES/GO MMMs performance on color removal of both synthetic melanoidin and spent wash effluent solution. 